\title{
Software Validation
}

National Cancer Institute

\section{Source}

National Cancer Institute. Software Validation. NCI Thesaurus. Code C142690.

A process of confirmation by examination and provision of objective evidence that software specifications conform to user needs and intended uses and that the particular requirements implemented through software can be consistently fulfilled. 